\title{
Vólvulo gástrico intratorácico y hernia diafragmática congénita de diagnóstico tardío. Informe de un caso
}

\author{
Dr. José Asz-Sigall, Dra. Karla Santos-Jasso, Dr. Silvio Carmona-Librado
}

\section{RESUMEN}

El vólvulo gástrico intratorácico es una complicación poco común de una hernia diafragmática, en la que el estómago sufre una torsión órganoaxial, que le predispone a estrangulación y necrosis y debe ser tratada como una urgencia quirúrgica ${ }^{1}$. Se informa el caso de un paciente con hernia diafragmática izquierda, complicada con vólvulo gástrico intratóracico. Se le operó con resultado satisfactorio.

Palabras clave: Vólvulo gástrico, hernia diafragmática, torsión órganoaxial, estrangulación, necrosis.

\section{ABSTRACT}

Intrathoracic gastric volvulus is a rare complication of diaphragmatic hernia, in which the stomach rotates in it's organoaxial axis which predisposes it to strangulation and necrosis. This condition should be treated promptly as a surgical emergency.

We report the case of a patient with a left diaphragmatic hernia complicated with an intrathoracic gastric volvulus which was successfully operated.

Keywords: Gastric volvulus, diaphragmatic hernia, organoaxial torsion, strangulation, necrosis.

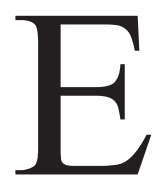

1 vólvulo gástrico es una anomalía de torsión poco frecuente, con una incidencia desconocida y con mortalidad de 42 a $56 \%$, causada por isquemia, perforación o necrosis del estómago ${ }^{2}$.

Las hernias diafragmáticas, la eventración diafragmática o el trauma torácico con ruptura diafragmática causan elongación de los ligamentos de la fijación gástrica y el vólvulo gástrico ${ }^{2,3}$. El uso creciente de estudios contrastados con bario para evaluar niños que sufren vómito y procesos respiratorios recurrentes, ha permitido incrementar el diagnóstico de las entidades predisponentes ${ }^{3}$.

Las manifestaciones clínicas del vólvulo gástrico son variables: desde la presentación aguda hasta síntomas

Instituto Nacional de Pediatría.

Correspondencia: Dr. José Asz-Sigall. Instituto Nacional de Pediatría. Insurgentes Sur 3700-C. Col. Insurgentes Cuicuilco. E-mail: jose asz@yahoo.com

Recibido: octubre, 2009. Aceptado: diciembre, 2009.

Este artículo debe citarse: Asz-Sigall J, Santos-Jasso K, CarmonaLibrado S. Vólvulo gástrico intratorácico y hernia diafragmática congénita de diagnóstico tardío. Informe de un caso. Acta Pediatr Mex 2010;31(1):32-35.

La versión completa de este artículo también está disponible en: www.nietoeditores.com.mx crónicos ${ }^{4}$. La suspicacia clínica y el estudio radiológico son esenciales para diagnosticar esta afección que pone en peligro la vida.

\section{CASO CLINICO}

Paciente masculino de 15 años previamente sano; inició su padecimiento actual tres días antes de su ingreso, con dolor abdominal en el epigastrio y el flanco izquierdo acompañado de náusea. Recibió antiespasmódicos que no le mejoraron. Un facultativo diagnosticó gastroenteritis y prescribió clonixinato de lisina y trimetropim-sulfametoxazol. El dolor abdominal se agravó. En estas condiciones ingresó a nuestra institución. El dolor abdominal era generalizado, el paciente tenía náusea y vómitos. Examen físico: Hipertensión arterial de 140/90. Ausencia de murmullo vesicular en el hemitórax izquierdo. En el abdomen no había datos de irritación peritoneal. Laboratorio: leucocitosis 15,300; neutrofilia 79\%, hipokalemia de $3.1 \mathrm{mEq} / \mathrm{L}$; gasometría, pH 7.44, $\mathrm{PO}_{2} 37 \mathrm{mmHg}$; $\mathrm{PCO}_{2} 28 \mathrm{mmHg}$; $\mathrm{HCO}_{3} 18.9 \mathrm{mmol} / \mathrm{L}$, déficit de base $-4.5 \mathrm{mmol} / \mathrm{L}$, lactato en $1.9 \mathrm{mmol} / \mathrm{L}$. Pruebas de funcionamiento hepático, pancreático, y química sanguínea normales. Depuración de creatinina $116 \mathrm{~mL} / \mathrm{m}^{2} \mathrm{sc} / \mathrm{min}$. 
Estudio radiológico. Se observó un nivel hidroáereo en el hemitórax izquierdo, había desplazamiento del mediastino a la derecha. No se pudo realizar una serie esófago-gastro-duodenal, debido a que el paciente tuvo una crisis convulsiva tónico clónica generalizada, que se controló con benzodiacepinas. Se optó por realizar una tomografía axial computada de cráneo que fue normal, tórax y abdomen simple y contrastada, que reveló hernia diafragmática izquierda y vólvulo gástrico. Figura (1 y 2).

El paciente se sometió a exploración quirúrgica. Se encontró una hernia diafragmática posterolateral izquierda que contenía estómago, colon, intestino delgado y bazo; el primero se hallaba muy dilatado; no se logró su reducción a través del orificio herniario a pesar de que se amplió a (8-10 $\mathrm{cm}$ aproximadamente) (Figura $3)$. Se efectuó descompresión gástrica por punción con aguja; se aspiraron $3.5 \mathrm{~L}$ de líquido gástrico. Se logró recolocar el estómago a la cavidad abdominal. Se reparó el defecto diafragmático con puntos en "U" de poliéster 0 , reforzados con malla de Goretex ${ }^{\circledR} \mathrm{Se}$ hizo una gastrostomía convencional tipo Stamm con sonda Pezzer 16Fr. El postoperatorio transcurrió en la unidad de cuidados intensivos, donde el paciente recibió ampicilina, amikacina, metronidazol y analgésicos, así como ventilación mecánica, y nutrición parenteral. Por el antecedente de crisis convulsivas recibió difenilhidantohína, y dexametasona con objeto de reducir el edema cerebral. Este problema se atribuyó a hipertensión arterial descontrolada, probablemente causada por compresión de los vasos renales o de la glándula suprarrenal izquierda. Se realizó una nueva TAC de cráneo 72 horas después de las crisis convulsivas con objeto de descartar isquemia cerebral; el estudio fue normal. Un electroencefalograma no mostró anormalidades, por lo cual cuatro días después se suspendió el fármaco antiepiléptico. Se extubó al $5^{\circ}$ día y evolucionó satisfactoriamente. La presión arterial se normalizó. El Servicio de Nefrología opinó que la hipertensión arterial fue de probable origen renovascular por compresión vascular y liberación de catecolaminas, ya que desapareció inmediatamente después de la operación. El paciente pudo alimentarse por vía oral al noveno día postquirúrgico; egresó a los 15 días; la sonda de gastrostomía se retiró un mes después de la cirugía. A los cuatro meses de evolución postquirúrgica el paciente permanece asintomático.

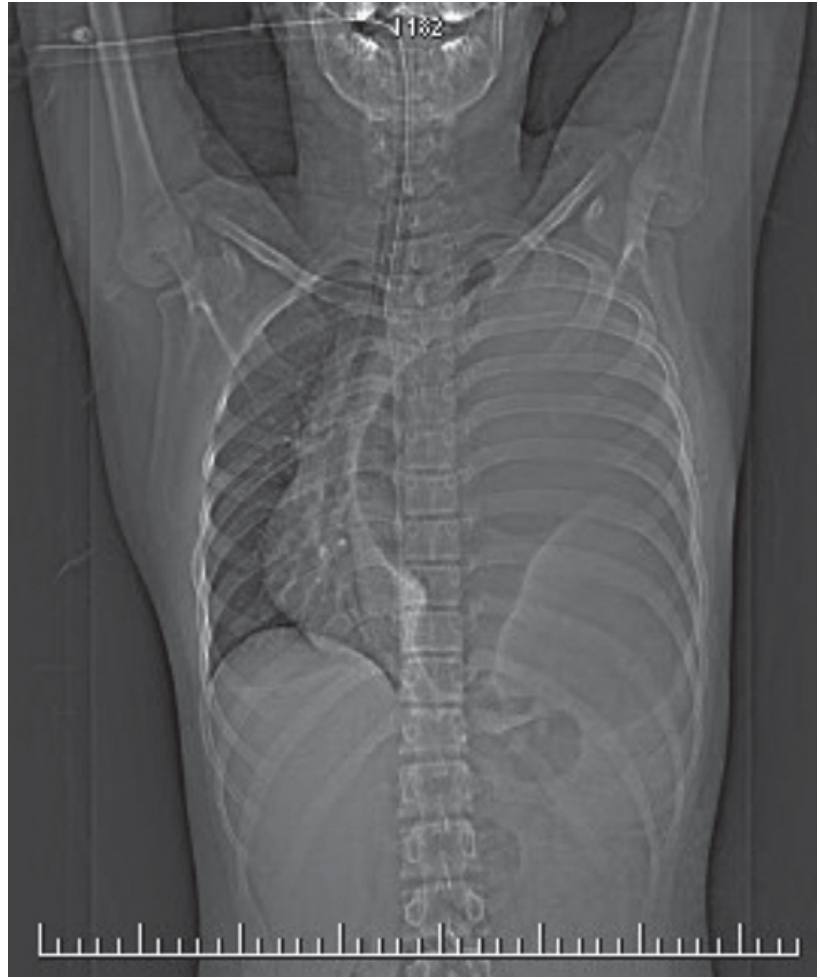

Figura 1. Topograma: vólvulo gástrico organo-axial.

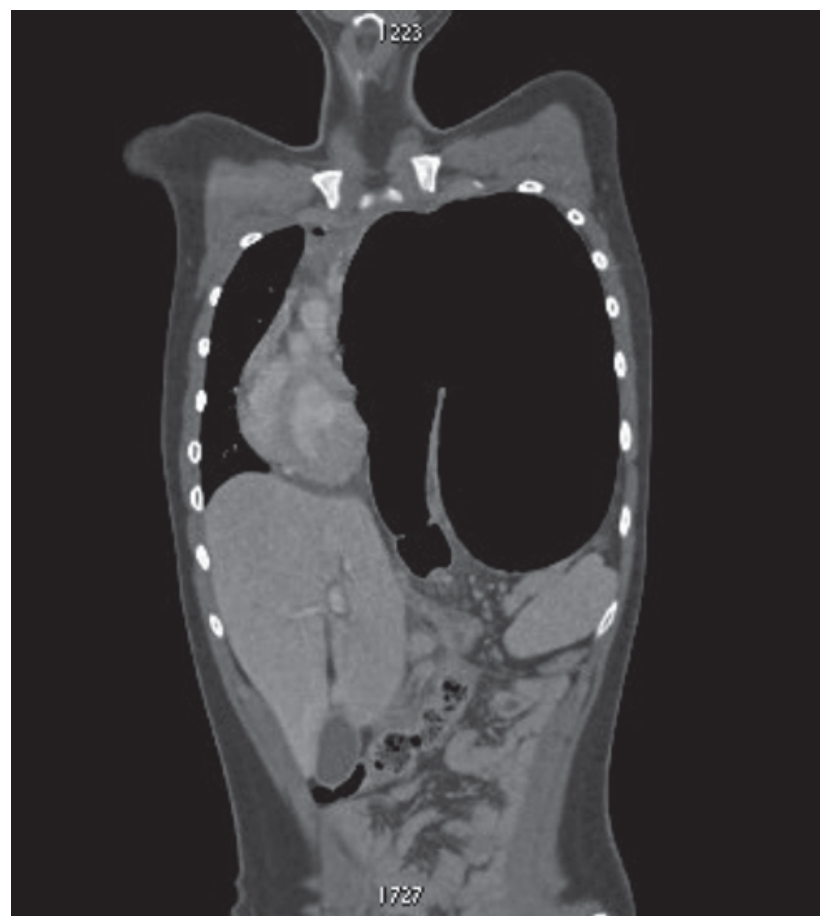

Figura 2. TAC de tórax que muestra una hernia diafragmática izquierda y vólvulo gástrico. 


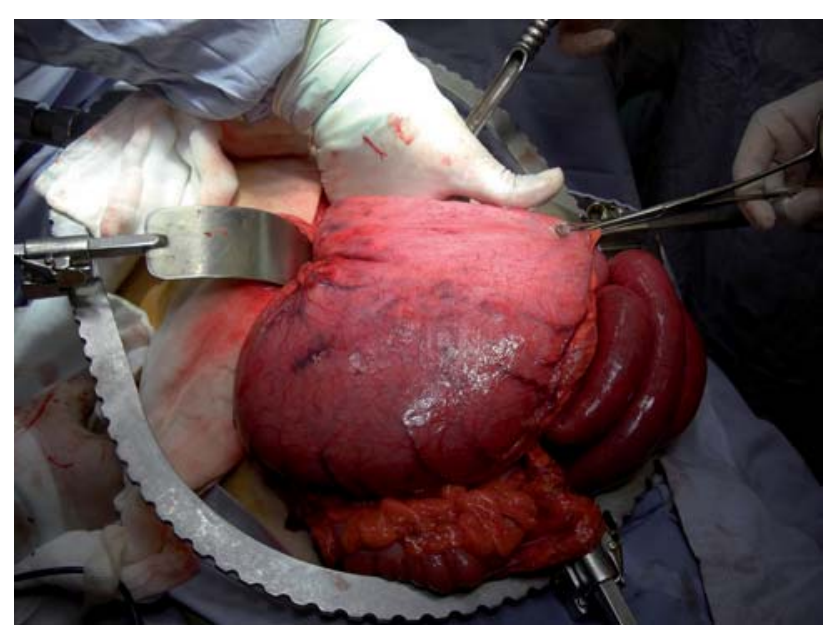

Figura 3. Persistencia gastromegalia posterior a la punción evacuadora para reducir la hernia diafragmática.

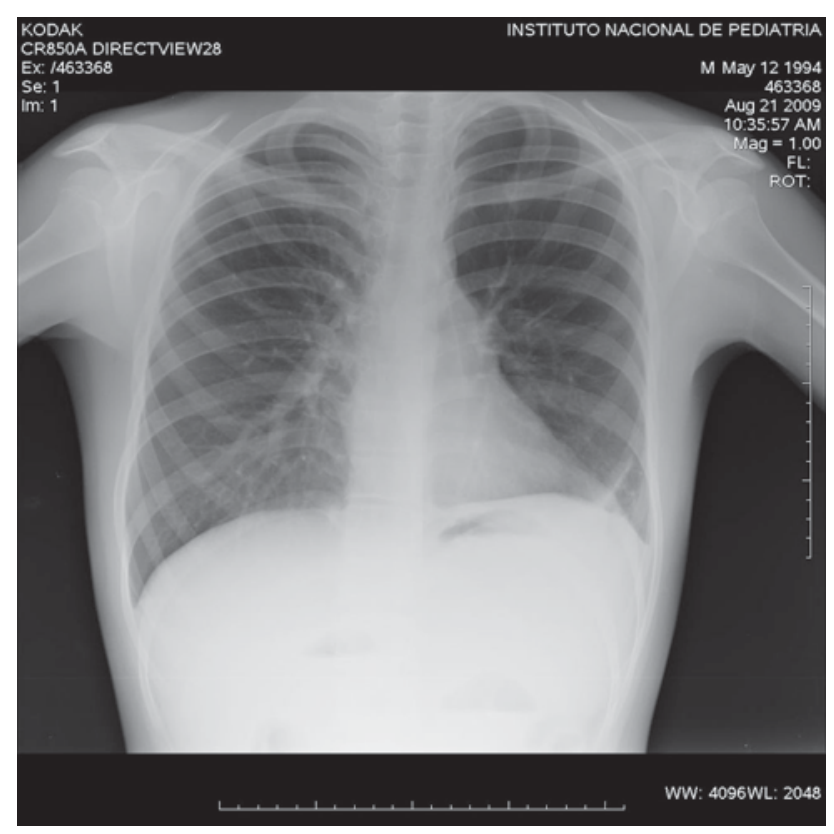

Figura 4. Placa simple de tórax; control post-quirúrgico. DISCUSION

La hernia diafragmática congénita puede presentarse en forma aguda en la etapa posnatal inmediata, con gran dificultad respiratoria lo que permite hacer el diagnóstico y efectuar una corrección quirúrgica temprana. Sin embargo, hay pacientes asintomáticos o que pueden presentar síntomas crónicos inespecíficos por meses o años en edades más avanzadas, incluso en la edad adulta, que se deben al desplazamiento de órganos intraabdominales al tórax y que permiten ser tratados de forma electiva, pero de manera rápida. En los casos de trauma toraco-abdominal severo ocurre ruptura diafragmática en un 5\% y en el 45 a $60 \%$ de estos se presenta la hernia de las vísceras abdominales al tórax. El órgano más frecuentemente herniado es el estómago, que puede sufrir una torsión órgano-axial que predisponga a su estrangulación y necrosis, lo que constituye una urgencia quirúrgica ${ }^{1,5}$. Hay informes de enfermos con vólvulo gástrico en casos de hernia hiatal y también como complicación postquirúrgica de una neumonectomía inferior izquierda, con elevación del diafragma ${ }^{6}$.

El término de vólvulo deriva del latin volveré, que significa vuelta o torcer. En 1579, Ambrose Paré describió el primer caso de la estrangulación gástrica por torsión ${ }^{7} \mathrm{de}-$ bido a una lesión del hemidiafragma izquierdo causada por una espada. Berti en 1866 describió la entidad clínica.

El vólvulo gástrico puede ser de presentación aguda ó crónica, $43 \%$ y $57 \%$ de los casos respectivamente, con diferentes manifestaciones clínicas ${ }^{8}$. El vólvulo primario es causado por la laxitud acentuada de los ligamentos de fijación del estómago: gastroesplénico, gastrofrénico, gastrocólico, y gastrohepático ${ }^{4}$. El vólvulo secundario se debe a anormalidades de la función gástrica, por ejemplo: distensión crónica causada por obstrucción del vaciamiento gástrico o por anormalidades de los órganos adyacentes, por ejemplo: anomalías diafragmáticas.

En los casos de vólvulo gástrico agudo, Cribbs y colaboradores hallaron en una revisión que hubo un porcentaje relativamente alto de asociación con anormalidades de vólvulo secundario en $69 \%$, sobre todo con anormalidades diafragmáticas (42\%).

En ocasiones el vólvulo gástrico es la primera presentación en un paciente asintomático con una anomalía diafragmática. A diferencia del anterior el vólvulo crónico generalmente es primario (74\%) y con menos frecuencia se acompaña de alguna anomalía $(26 \%)^{9}$.

Según el eje de rotación del órgano, el vólvulo puede ser órgano-axial en 59\% de los casos ${ }^{2}$, con rotación alrededor de su eje cardiopilórico o del eje longitudinal, que se extiende desde la unión gastroesofágica hasta el píloro ${ }^{8,10,11}$. La curvatura mayor del estómago se desplaza de izquierda a derecha y de atrás hacia delante para formar el vólvulo.

Otro tipo de vólvulo es el llamado mesentero-axial (29\%): En este caso el estómago sufre una rotación alrededor del eje imaginario que va desde el centro de su curvatura mayor hasta el "porta hepatis" (hilio hepático). En este caso se expresa clínicamente en forma aguda, 
intermitente o crónica ${ }^{10}$. En $12 \%$ de los casos pueden combinarse ambos tipos (órgano-axial y mesenteroaxial) ${ }^{2,8}$, tipo mixto.

Clásicamente, la tríada de Borchardt, (1904) consiste en vómitos, distensión epigástrica y dificultad para el paso de un tubo nasogástrico; su presencia debe hacer pensar en un vólvulo gástrico como primer diagnóstico. Esta tríada ocurre en $70 \%$ de los casos. La inestabilidad hemodinámica y el mal estado general no siempre están presentes. Los estudios retrospectivos muestran que la presentación común de vólvulo gástrico crónico es con disfagia, dolor epigástrico y dolor torácico, lo que ocurre en un $29 \%$ de los casos. La presencia de una hernia hiatal que curse con vómitos persistentes a pesar de tratamiento antiemético, debe hacer sospechar en un vólvulo gástrico aunque el paciente parezca estable. El estudio radiológico algunas veces muestra una gran o una doble burbuja de gas con nivel hidroaéreo en el cuadrante superior izquierdo del abdomen. Las elevaciones de hemidiafragma izquierdo hacen sospechar una eventración diafragmática. Una serie esófago-gastro-duodenal permite hacer el diagnóstico. La TAC puede no ser necesaria pero proporciona datos que apoyan el diagnóstico.

Una vez hecho el diagnóstico, el siguiente paso es el tratamiento quirúrgico. La elección del abordaje quirúrgico depende del tiempo de evolución de la afección. Se prefiere operar por la vía torácica en el paciente crónico, debido a la presencia probable de adherencias. En los casos agudos, la vía abdominal es la más recomendada para tratar probables lesiones de los órganos intraabdominales. Puede emplearse cirugía abierta o de mínima invasión. En los casos de vólvulo, la morbilidad aumenta y debe considerarse la posibilidad de gastrectomía total o parcial, según los hallazgos quirúrgicos transoperatorios ${ }^{1}$. Siempre debe dejarse un buen drenaje gástrico post-quirúrgico como una gastrostomía descompresiva, con objeto de evitar la distensión gástrica. Se debe recomendar siempre la reparación del defecto diafragmático, y en los casos de eventración o vólvulo primario una gastropexia. ${ }^{8}$

\section{CONCLUSIONES}

La presencia de una hernia diafragmática izquierda predispone a la herniación del estómago con vólvulo agudo o crónico. El diagnóstico en casos crónicos debe sospecharse por el antecedente de síntomas inespecíficos como dolor abdominal epigástrico, sensación de saciedad temprana, reflujo gastroesofágico y vómitos gástricos, estreñimiento, diarrea o ambos. El estudio radiológico simple sugiere fuertemente el diagnóstico, y el estudio contrastado del esófago, estómago y duodeno confirma la sospecha. El tratamiento quirúrgico es una urgencia, que puede abordarse por laparoscopia o por cirugía abierta; la cual incluye descompresión gástrica ${ }^{1}$, corrección del vólvulo, reducción de vísceras abdominales del compartimento torácico, reducción o resección del saco, corrección del defecto diafragmático ${ }^{5}$, y gastropexia a la pared abdominal anterior con la colocación de un tubo de gastrostomía para evitar vólvulo recurrente ${ }^{2}$. La intervención del equipo médico en forma oportuna, mejora el pronóstico de estos pacientes ${ }^{7}$.

\section{BIBLIOGRAFÍA}

1. Ospino SG, Martínez BP, Valverde RR, Vólvulo gastric intratorácico secundario a hernia diafragmática izquierda crónica postraumática. Acta Méd. Costarric. 2005;47(2):94-6.

2. Chau B, Dufel S. Gastric volvulus.Emerg Med J. 2007;24(6): 446-7.

3. Al-Salem AH. Acute and chronic gastric volvulus in infants and children: who should be treated surgically? Pediatr Surg Int. 2007;23(11):1095-9.

4. Mayo A, Erez I, Lazar L, Rathaus V, Konen O, Freud E. Volvulus of the stomach in childhood: the spectrum of the disease. Pediatr Emerg Care. 2001;17(5):344-8.

5. Katkhounda N, Mavor E, Achanta K, Friedlander M, Grant S, Essani R, et al. Laparoscopic repair of chronic intrathoracic gastric volvulus. Surgery. 2000;128:784-90.

6. Fevzi B, Uygur-Bayramicli O, Guler S, Yildizeli B, Yuksel M. Laparoscopic repair of a gastric volvulus occurring as a longterm complication of left pneumonectomy: report of a case. Surg Today. 2007;37:43-5.

7. Cabrera TMG, Renedo RJL, Tejeda THD. Vólvulo gástrico. Informe de un caso. Acta Pediatr Mex. 2009;30(3):163-6.

8. Gerstle JT, Chiu P, Emil S. Gastric volvulus in children: lessons learned from delayed diagnoses. Sem Ped Surg. 2009;18: 98-103.

9. Cribbs RK, Gow KW, Wulkan M. Gastric volvulus in infants and children. Pediatrics. 2008;122:752-62.

10. Goonetillke G. Chronic volvulus of the stomah with eventration of the diafragm. Ceylon Med J. 1995;40:153-9.

11. Lee JS, Park JW, Sohn JW, Kim KC, Hwang SG, Park PW, et al. Organoaxial volvulus of the stomach with diaphragmatic eventration. Korean J Intern Med. 2000; 15 (2):127-30.

12. Channer Lt, Squires GT, Price PD. Laparoscopic repair of gastric volvulus. JSLS. 2000;4(3):225-30. 